\title{
ADAPTIVE INVERSE CONTROL OF PIEZOELECTRIC ACTUATORS WITH HYSTERESIS OPERATORS
}

\author{
K. Kuhnen, H. Janocha \\ Laboratory for Process Automation (LPA) \\ University of Saarland, Im Stadtwald, Geb. 13 \\ D-66123 Saarbrücken, Germany \\ fax: +49-681-302-2678 and e-mail:klaus@1pa.uni-sb.de
}

Keywords: adaptive control, nonlinear control, hysteresis, piezoelectric actuator

\begin{abstract}
The present paper will describe an approach for the compensation of the hysteretic transfer characteristics of a piezoelectric stack transducer by an adaptive inverse hysteretic control. The basis of the inverse control is formed by a weighted superposition of elementary stop-type hysteresis operators which, in terms of mathematics, can be easily described and which reflect the qualitative properties of the inverse transfer characteristic of the transducer. Starting with a linear characteristic the weigths of an inverse hysteretic observer are identified during operation by a stable adaption law and transformed to the controller parameter. As a result the maximum linearity error caused by hysteresis is lowered about one order of magnitude.
\end{abstract}

\section{Introduction}

Because of its high dynamic and nearly unlimited resolution piezoelectric actuators are best suited as drives in micropositioning systems. To guarantee a high precision positioning under variable operating conditions the position is normally controlled in a closed loop with the help of an high precision position sensor and a PID-controller [2]. With the assumption of a linear transfer characteristic of the transducer the controller guarantees a fast and sufficiently damped transient over the whole operating range, if the parameters of the controller are well adjusted. However, the assumption of a linear transfer characteristic of the transducer is only a crude approximation because the transducer is driven with highvoltage amplitudes to generate the longest possible displacements. Due to this high-voltage modulation, the electromechanical transfer characteristic is considerably characterized by rate-independent hysteresis effects shown in Fig. 1. This leads to a reduction of the control performance in the large signal operating range [6].

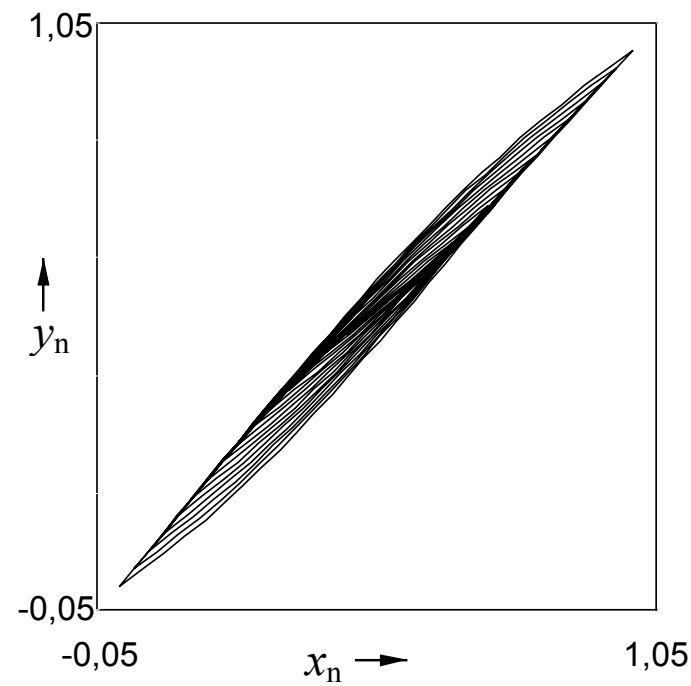

Fig 1: Normalized electromechanical transfer characteristic of a piezoelectric stack transducer

The present paper will describe an alternative solution for the compensation of the hysteretic transfer characteristics of a piezoelectric stack transducer by an adaptive inverse hysteretic control. For that purpose the real hysteretic transfer characteristic of the transducer is modeled by a complex hysteresis operator $H[]$. Based on this hysteresis operator an inverse hysteresis operator $H^{-1}[]$ is derived, which can be used as a feed-forward compensator, see Fig.2 [7].

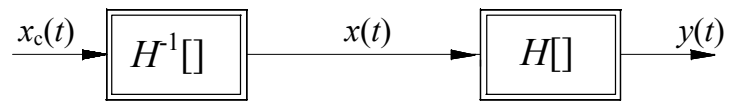

Fig 2: Signal flow chart of the inverse control

In this approach the model parameter of the inverse hysteretic control is determined on-line during the operation of the transducer. By this means a self-learning or adaptive hysteretic inverse control can be realized. 


\section{Theoretical foundations}

In the mathematical literature the notation of the hysteretic nonlinearity is equated with the notation "rate independent memory effect" [8]. This means that the output signal of a system with hysteresis depends not only on the present value of the input signal but also on the order of their amplitudes, especially their extremum values, but not on their rate in the past. The rate-independent branching transfer characteristic shown in Fig. 1 is typical for a system with hysteretic nonlinearities. Because of its phenomenological character the concept of hysteresis operators developed by Krasnosel'skii and Pokrovskii in the 1970s allows a general and precise modeling of hysteretic system characteristic [3]. The basic idea consists in the modeling of the real hysteretic transfer characteristic by the weighted superposition of many elementary hysteresis operators, which differ according to the type of the elementary operator in one or more characteristic parameters. Two of the simplest types of such elementary hysteresis operators are the so-called linear-play operator (LPO)

$$
y(t)=p_{\mathrm{r}}[x](t)
$$

and the so called linear-stop operator (LSO)

$$
s_{\mathrm{r}}[x](t)=x(t)-p_{\mathrm{r}}[x](t)
$$

[1]. $x(t)$ is the input-signal and $y(t)$ is the output-signal of the elementary hysteretic system. Fig. 3 and Fig. 4 show the rateindependent transfer characteristic of the LPO and the LSO in an $y(x)$-representation.

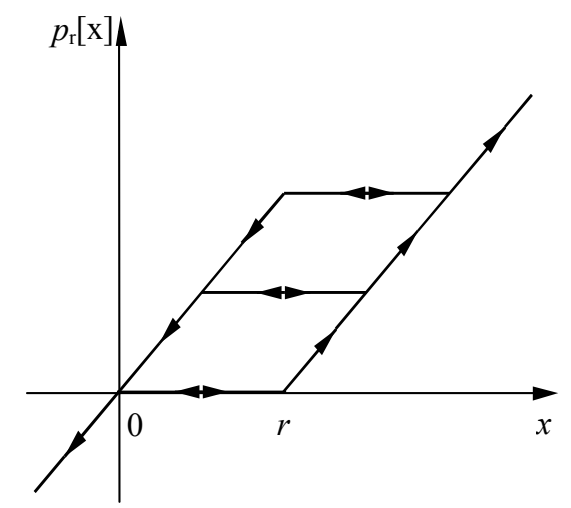

Fig. 3: Rate-independent transfer characteristic of an LPO

Both operators are characterized by its threshold parameter $r$. For the precise modeling of real hysteresis phenomena several elementary hysteresis operators with different threshold values $r_{\mathrm{i}}$ can be superimposed. This parallel connection of elementary hysteresis operators leads to the so-called discrete Prandtl-Ishlinskii operator of play-type (PIOP)

$$
y(t)=P[x](t)=\sum_{\mathrm{i}=1}^{\mathrm{n}} q_{\mathrm{i}} \cdot p_{\mathrm{r}_{\mathrm{i}}}[x](t)=\boldsymbol{p}_{\boldsymbol{r}}^{\mathrm{T}}[x](t) \cdot \boldsymbol{q}
$$

and to the so-called discrete Prandtl-Ishlinskii operator of stop-type (PIOS) [1]

$$
y(t)=S[x](t)=\sum_{\mathrm{i}=1}^{\mathrm{n}} a_{\mathrm{i}} \cdot s_{\mathrm{r}_{\mathrm{i}}}[x](t)=\boldsymbol{s}_{\boldsymbol{r}}^{\mathrm{T}}[x](t) \cdot \boldsymbol{a} .
$$

In practice, due to the continuity of the linear-play and the linear-stop operator, complex hysteresis loops can be modeled in a sufficiently precise way with the help of a small number of elementary operators [4]. Therefore PIOP and PIOS are suitable tools for the real-time calculation of the complex hysteresis transfer characteristics.

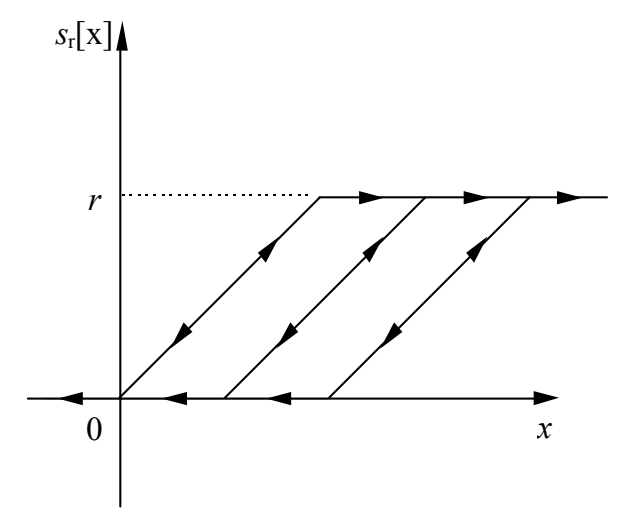

Fig. 4: Rate-independent transfer characteristic of an LSO

There is a specific relationship between the PIOP and the PIOS, which is fundamental for control applications. If the PIOP is a strictly monotone hysteresis operator, it was shown in [1] that there exists a strictly monotone PIOS with

$$
y(t)=P\left[S\left[x_{\mathrm{c}}\right]\right](t)=I d\left[x_{\mathrm{c}}\right](t)=x_{\mathrm{c}}(t) .
$$

Here $I d[]$ is the identity operator. As a consequence of equation (5) a hysteretic inverse control $H^{1}[]$ can be developed by a PIOS, if the transducer $H[]$ can be modeled in a sufficiently precise way by a PIOP and vice versa.

\section{Adaptive inverse control}

To realize an adaptive inverse hysteretic control for a complex hystereric plant the following assumptions must be fulfilled.

- The hysteretic plant is strictly monotone and can be described by a PIOP or a PIOS. 
- The input amplitude range $\left[x_{\min }, x_{\max }\right]$ and the output amplitude range $\left[y_{\min }, y_{\max }\right]$ of the hysteretic plant are bounded and known.

In the case of a voltage driven piezoelectric stack transducer a PIOP is suited to describe its hysteretic transfer characteristic [4] and so the inverse control can be built up by a PIOS. For the inverse control of the hysteretic plant both the threshold parameters $r_{\mathrm{i}}$ and the weights $a_{\mathrm{i}}$ of the PIOS must be known. Because of the difficult identification of the threshold parameters during operation the input and output amplitude range of the plant will be mapped on the unity range $[0,1]$ by a linear transformation rule and the threshold parameters will be set in the range ]0,1]. The distribution of the threshold values in the range $] 0,1]$ is oriented on the curvature of the major hysteretic loop. In the case of a constant curvature, for example, a normal distribution is suitable. Due to this procedure the transfer characteristic of the LSO is completely determined and the transfer characteristic of the PIOS is only linearly dependent on the weights. Thus the control equation is given by

$$
x_{\mathrm{n}}(t)=S_{\mathrm{c}}\left[x_{\mathrm{nc}}\right](t)=\boldsymbol{s}_{\mathrm{c}}^{\mathrm{T}}\left[x_{\mathrm{nc}}\right](t) \cdot \boldsymbol{a}_{\mathrm{c}}(t)
$$

Here $x_{\mathrm{nc}}(t)$ is the normalized control signal, $x_{\mathrm{n}}(t)$ the normalized inverse control or input signal of the plant and $\boldsymbol{a}_{\mathrm{c}}$ the vector of weights of the inverse control. From the equations (5) and (7) follows that the connection between the normalized output signal $y_{\mathrm{n}}(t)$ and the normalized input signal $x_{\mathrm{n}}(t)$ of the plant can also be described by a PIOS

$$
x_{\mathrm{n}}(t)=S_{\mathrm{p}}\left[y_{\mathrm{n}}\right](t)=\boldsymbol{s}_{\mathrm{p}}^{\mathrm{T}}\left[y_{\mathrm{n}}\right](t) \cdot \boldsymbol{a}_{\mathrm{p}}(t)
$$

which models the inverse hysteretic transfer characteristic of the plant. Thus the basic idea for the realization of an adaptive inverse hysteretic control consists in two steps. First, an adaptive inverse hysteretic observer for the weights $\boldsymbol{a}_{\mathrm{p}}(t)$ will be realized in such a manner that all signals in the system are bounded and the normalized observation error

$$
e_{\mathrm{o}}(t)=x_{\mathrm{n}}(t)-\boldsymbol{s}_{\mathrm{o}}^{\mathrm{T}}\left[y_{\mathrm{n}}\right](t) \cdot \boldsymbol{a}_{\mathrm{o}}(t)
$$

tends to zero asymptotically. In equation (9) $\boldsymbol{a}_{\mathrm{o}}(t)$ stands for the estimates of the plant parameters $\boldsymbol{a}_{\mathrm{p}}(t)$. Secondly, a transformation of the observed parameters $\boldsymbol{a}_{\mathrm{o}}(t)$ to the control parameters $\boldsymbol{a}_{\mathrm{c}}(t)$ will be carried out in such a manner that all signals in the system remain bounded under the influence of the inverse control and the normalized control error

$$
e_{\mathrm{c}}(t)=x_{\mathrm{nc}}(t)-y_{\mathrm{n}}(t)
$$

tends to zero asymptotically. Assuming a bounded normalized input and output signal within the amplitude range $[0,1]$ an adaption law for the weights of the inverse hysteretic observer is given by

$$
d\left[\boldsymbol{a}_{\mathrm{o}}\right](t)=\gamma \cdot e_{\mathrm{o}}(t) \cdot \boldsymbol{s}_{\mathrm{o}}\left[y_{\mathrm{n}}\right](t)
$$

with the differential operator $d[]$. This rule guarantees that the weights are bounded and the observation error tends to zero asymptotically [5]. $\gamma$ is an adaptive gain which suitable chosen increases the learning performance of the adaptive inverse hysteretic observer.

To develop a suited transformation rule for the inverse controller parameter the following considerations can be made. If the observer is driven with the maximum normalized output amplitude $y_{\text {nmax }}=1$ as a reaction of the plant to a maximum normalized input amplitude $x_{\mathrm{n} \max }=1$ then

$$
x_{\mathrm{omax}}=\boldsymbol{s}^{\mathrm{T}}[1] \cdot \boldsymbol{a}_{\mathrm{o}}(t)=\boldsymbol{r}^{\mathrm{T}} \cdot \boldsymbol{a}_{\mathrm{o}}(t)
$$

holds for a strictly monotone observer. Due to the limitation of the normalized input and output signal in the range $[0,1]$ the adaption law (11) leads to

$$
\lim _{\mathrm{t} \rightarrow \infty}\left(\boldsymbol{s}^{\mathrm{T}}\left[y_{\mathrm{n}}\right](t) \cdot \boldsymbol{a}_{\mathrm{o}}(t)\right)=\lim _{\mathrm{t} \rightarrow \infty} x_{\mathrm{o}}(t)=x_{\mathrm{n}}(t)
$$

and thus

$$
\lim _{\mathrm{t} \rightarrow \infty}\left(\boldsymbol{r}^{\mathrm{T}} \cdot \boldsymbol{a}_{\mathrm{o}}(t)\right)=\lim _{\mathrm{t} \rightarrow \infty} x_{\mathrm{omax}}=x_{\mathrm{nmax}}=1
$$

holds. If the normalized control signal $x_{\mathrm{nc}}(t)$ is also assumed to be bounded within the range $[0,1]$, the transformation rule

$$
\boldsymbol{a}_{\mathrm{c}}(t)=\frac{\boldsymbol{a}_{\mathrm{o}}(t)}{\boldsymbol{r}^{\mathrm{T}} \cdot \boldsymbol{a}_{\mathrm{o}}(t)}
$$

guarantees a bounded normalized inverse control or input signal $x_{\mathrm{n}}(t)$ within the range [0,1], regardless of the specific values of the observer weights $\boldsymbol{a}_{\mathrm{o}}$ (here the singular case $\boldsymbol{a}_{\mathrm{o}}(t)$ $=\mathbf{0}$ is excluded). But exactly this was the assumption for a stable determination of the observer weights $\boldsymbol{a}_{\mathrm{o}}$. From equation (14) and (15) follows

$$
\lim _{t \rightarrow \infty} \boldsymbol{a}_{\mathrm{c}}(t)=\boldsymbol{a}_{\mathrm{o}}(t)
$$

Hence the controller tends asymptotically to the same inverse hysteretic transfer characteristic as the observer and with equation (5) the control error (10) tends to zero asymptotically, too. Fig. 5 shows the continous time signal flow chart of the adaptive inverse control. In this Figure the blocks $T_{\mathrm{x}}$ and $T_{\mathrm{y}}$ stand for the transformation of the input and output signal in the unity range $[0,1]$.

The block $T_{\mathrm{x}}^{-1}$ is the inverse linear transformation to $T_{\mathrm{x}}$ and $T_{\boldsymbol{a}}$ stands for the nonlinear parameter transformation (15). For the implementation of the adaptive inverse control on a digital signal processor (DSP) the LSO was replaced by the discrete time LSO 


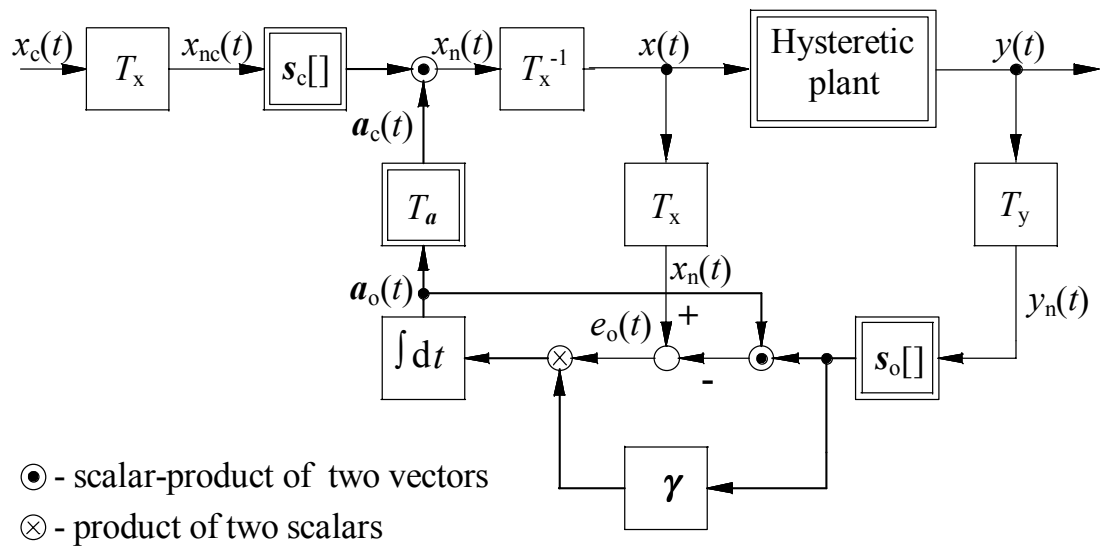

Fig 5: Signal flow chart of the adaptive inverse hysteretic controller

with

$$
s_{\mathrm{r}}[x](k)=x(k)-\eta_{\mathrm{r}}(k)
$$

$$
\eta_{\mathrm{r}}(k)= \begin{cases}x(k), & \text { if } \eta_{\mathrm{r}}(k-1)>x(k) \\ \eta_{\mathrm{r}}(k-1), & \text { if } \eta_{\mathrm{r}}(k-1) \leq x(k) \leq \eta_{\mathrm{r}}(k-1)+r \\ x(k)-r, & \text { if } \eta_{\mathrm{r}}(k-1)+\mathrm{r}<x(k)\end{cases}
$$

and the differential operator by the difference operator

$$
d[x](k)=\frac{x(k+1)-x(k)}{T_{\mathrm{s}}} .
$$

$T_{\mathrm{s}}$ is the sampling time of the process. The replacement of the hysteresis and differential operators by their discrete time counterparts leads to the following discrete time inverse observer equation

$$
\begin{aligned}
\boldsymbol{a}_{\mathrm{o}}(k+1) & =\left(\boldsymbol{I}-\gamma \cdot T_{\mathrm{s}} \cdot \boldsymbol{s}_{\mathrm{o}}\left[y_{\mathrm{n}}\right](k) \cdot \boldsymbol{s}_{\mathrm{o}}^{\mathrm{T}}\left[y_{\mathrm{n}}\right](k)\right) \cdot \boldsymbol{a}_{\mathrm{o}}(k) \\
& +\gamma \cdot T_{\mathrm{s}} \cdot \boldsymbol{s}_{\mathrm{o}}\left[y_{\mathrm{n}}\right](k) \cdot x_{\mathrm{n}}(k)
\end{aligned}
$$

transformation rule

$$
\boldsymbol{a}_{\mathrm{c}}(k)=\frac{\boldsymbol{a}_{\mathrm{o}}(k)}{\boldsymbol{r}^{\mathrm{T}} \cdot \boldsymbol{a}_{\mathrm{o}}(k)},
$$

and the inverse controller equation

$$
x_{\mathrm{n}}(k+1)=\boldsymbol{s}_{\mathrm{c}}^{\mathrm{T}}\left[x_{\mathrm{nc}}\right](k) \cdot \boldsymbol{a}_{\mathrm{c}}(k)
$$

The difference in the inverse controller equation results from the sample and hold operation of the DSP-based controller.

\section{Results}

After the development of the adaptive inverse feed forward control its performance has been tested on a real multilayer stack transducer. For this purpose the inverse hysteretic transfer characteristic of a voltage-driven piezoelectric stack transducer has been modeled by a PIOS with 10 LSOs. The threshold values of the PIOS are normal distributed in the amplitude range ]0,1] and remain fixed during operation. Tab. 1 shows the threshold values and the initial values of the observer and controller weights. Within the range $[0,1]$ the LSO with the threshold value $r=1.0$ corresponds to the identity operator $I d[]$. Therefore, both the controller and the observer have a transfer characteristic of an identity operator at start time.

\begin{tabular}{|c|c|c|c|}
\hline Number & $r_{\mathrm{i}}$ & $a_{\mathrm{oi}}$ & $a_{\mathrm{ci}}$ \\
\hline 1 & 0.1 & 0.0 & 0.0 \\
\hline 2 & 0.2 & 0.0 & 0.0 \\
\hline 3 & 0.3 & 0.0 & 0.0 \\
\hline 4 & 0.4 & 0.0 & 0.0 \\
\hline 5 & 0.5 & 0.0 & 0.0 \\
\hline 6 & 0.6 & 0.0 & 0.0 \\
\hline 7 & 0.7 & 0.0 & 0.0 \\
\hline 8 & 0.8 & 0.0 & 0.0 \\
\hline 9 & 0.9 & 0.0 & 0.0 \\
\hline 10 & 1.0 & 1.0 & 1.0 \\
\hline
\end{tabular}

Tab. 1: Threshold values of the PIOS and the corresponding initial values of the observer weights

The product of the sample time $T_{\mathrm{s}}$ and the adaptive gain $\gamma$ was chosen to 0.5. Fig. 6 shows the learning process of the controller, if it was periodically excited with a normalized control signal shown in Fig. 7. 

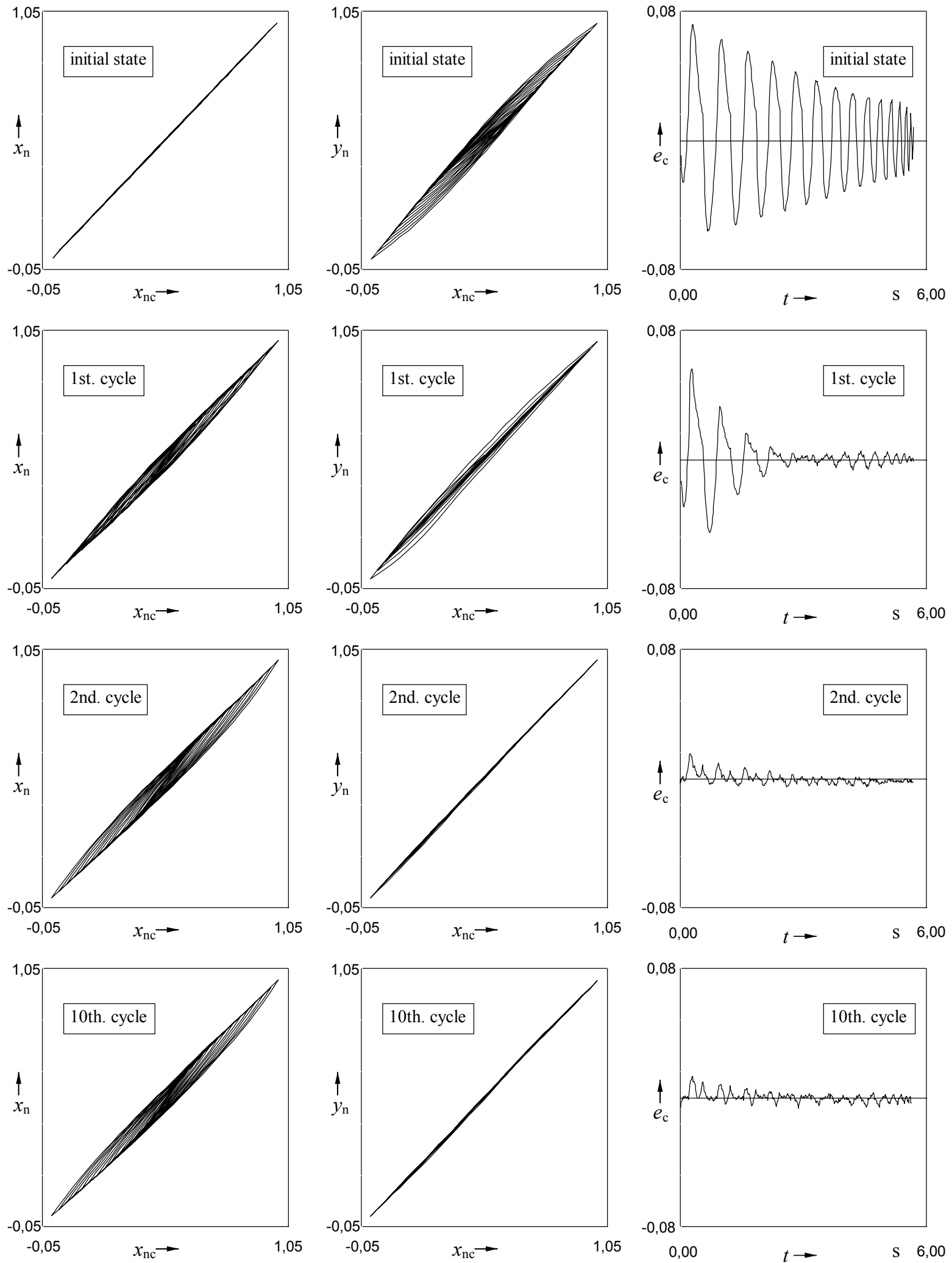

Fig. 6: Measured learning process of the inverse control 


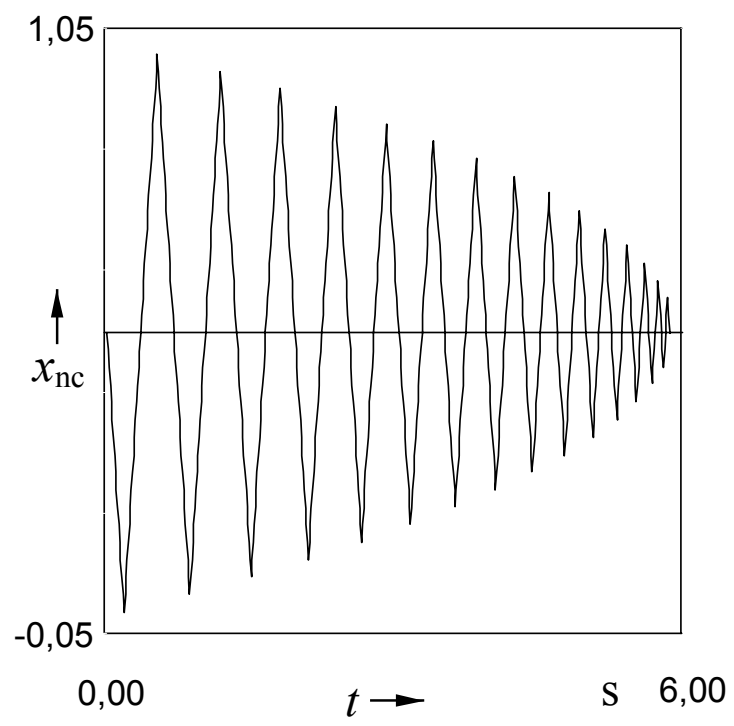

Fig 7: Normalized control signal for the performance test of the inverse controller.

The first column in Fig. 6 shows the rate independent transfer characteristic of the inverse controller, the second column the serial combination of the inverse controller and the real transducer and the third column the normalized control error over time. The first row in Fig. 6 shows the transfer characteristic at the start time. The second, third and fourth row of Fig. 6 show the transfer characteristics after the first, second and tenth learning cyle. During the first cycle the operation of the controller is still imperfect. But the overall linearity error is already at this time smaller than in the operation of the transducer without the controller, see the first row of Fig. 6. After the second learning cycle the transfer characteristic of the controller is nearly the inverse of the transducer and therefore the serial combination is nearly linear. Because of the imperfection of the inverse hysteresis model, after the second cycle no improvement of the transfer characteristic worth mentioning can be reached, see the fourth row in Fig. 6. With the adaptive inverse hysteretic control the normalized control error is reduced to a maximum value of about $1 \%$ in comparison to the maximum value of about $8 \%$ without the inverse control.

\section{Summary and prospects}

Measurements on a real piezoelectric transducer have shown that an adaptive inverse control for the compensation of the hysteretic transfer characteristic of a piezoelectric stack transducer can be built up by a stop-type Prandtl-Ishlinskii operator if the transducer can be modeled by a play-type Prandtl-Ishlinskii operator. For the adaption of the inverse control on the real hysteretic plant an inverse hysteretic observer was constructed by a stop-type Prandtl-Ishlinskii operator which was coupled with a stable adaption law for the on-line identification of the observer parameter. The controller parameters follow from the observer parameters by a nonlinear transformation rule which guarantees the stability and convergence of the overall system to an identity operator. In future works the method will be extended to plants which consist of a rate-independent hysteretic characteristic followed by a LTI-system which models the mechanical surroundings of the hysteretic actuator in real applications.

\section{Acknowledgements}

The authors thank the Deutsche Forschungsgemeinschaft (DFG) for the financial support of this work.

\section{References}

[1] Brokate M., Sprekels J., "Hysteresis and Phase Transitions", Springer-Verlag, Berlin Heidelberg New York, (1996).

[2] Hicks T.R., Atherton P.D., "The NanoPositioning Book", Queensgate Instruments Ltd. (1997)

[3] Krasnosel'skii M. A., Pokrovskii A. V., "Systems with Hysteresis". Springer-Verlag, Berlin, (1989).

[4] Kuhnen K., Janocha H., "Compensation of the Creep and Hysteresis Effects of Piezoelectric Actuators with Inverse Systems", 6th International Conference on New Actuators, Bremen, 309-312, (1998)

[5] Narendra K.S., Annaswamy A.M., "Stable Adaptive Systems", Prentice-Hall Inc., New Jersey, (1989)

[6] Ping G., Musa J.,"Generalized preisach model for hysteresis nonlinearity of piezoceramic actuators", Precision Engineering, 20, 99-111, (1997)

[7] Schäfer J., Janocha H., "Compensation of hysteresis in solid state actuators", Sensors and Actuators, A49, 97102, (1995)

[8] Visintin A., "Differential Models of Hysteresis", SpringerVerlag, Berlin Heidelberg New York, (1996) 